\title{
Structure Evolution in a Polyurea Segmented Block Copolymer Because of Mechanical Deformation
}

\author{
J. A. Pathak, ${ }^{\dagger,}$ J. N. Twigg, ${ }^{\ddagger}$ K. E. Nugent, ${ }^{\ddagger}$ D. L. Ho, ${ }^{\S}$ E. K. Lin, ${ }^{\S}$ P. H. Mott, ${ }^{\ddagger}$ \\ C. G. Robertson, ${ }^{\|}$M. K. Vukmir, ${ }^{\perp}$ T. H. Epps III, ${ }^{\perp}$ and C. M. Roland ${ }^{*, *}$ \\ Chemistry \& Biochemistry Department, George Mason University, Fairfax, Virginia 22030-4444; \\ Chemistry Division, Code 6120, US Naval Research Laboratory, Washington, D.C. 20375-5342; \\ Polymers Division, National Institute of Standards and Technology, Mail Stop 8541, Gaithersburg, \\ Maryland 20899-8541; Northboro Research and Development Center, Saint-Gobain High-Performance \\ Materials, Northboro, Massachusetts 01532-1545; and Chemical Engineering Department, University of \\ Delaware, Colburn Laboratory, Newark, Delaware 19716
}

Received May 16, 2008; Revised Manuscript Received August 1, 2008

\begin{abstract}
Extensional stress-strain measurements on a polyurea (PU) were carried out at strain rates up to $830 \mathrm{~s}^{-1}$, in combination with ex post facto small-angle X-ray scattering (SAXS) measurements and temperaturedependent SAXS. The elastomer is of interest because of its application as an impact-resistant coating. The highest strain rates used herein fall within the softening, or transition, zone of the viscoelastic spectrum and are thus relevant to the working hypothesis that the performance of a polyurea impact coating is related to its transition to the glassy state when strained very rapidly. While quasi-static and slow deformation of the PU gives rise to irrecoverable strain and anisotropic SAXS patterns, when stretched at high rates the PU recovers completely and the scattering is isotropic. Thus, the deformation of the hard domains observed at low rates is absent at high strain rates. Linear dynamic mechanical measurements were also carried out, with the obtained segmental relaxation times in good agreement with dielectric relaxation measurements on this material. The PU exhibits the usual breakdown of time-temperature superposition in the transition zone. This thermorheological complexity underlies the fact that published time-temperature shift factors for this material are unrelated to the segmental dynamics, and therefore use of these shift factors to predict the onset of glassy dynamics during impact loading of the PU will be in error.
\end{abstract}

\section{Introduction}

There is substantial interest in the effect of high strain rates on polyurea (PU) due to its use as an impact-resistant coating. ${ }^{11}$ The properties of PU make it an attractive candidate for this type of application: The reaction of isocyanate with polyetheramine to form the material is very rapid, so that the coating properties are largely independent of ambient temperature and humidity. The microphase-separated segmented block copolymer morphology consists of hard segment domains dispersed in a soft matrix. ${ }^{12-14}$ The hard domains are extensively hydrogen-bonded ${ }^{15,16}$ and function as both reversible physical cross-links and a reinforcing filler, thus providing good mechanical properties, especially toughness. ${ }^{17-20}$ Mechanisms proposed for the enhanced performance of PU-coated substrates include a delay in the onset of necking in the substrate, ${ }^{1}$ an impact-induced transition to a glassy state of the PU soft segments, ${ }^{2}$ and retardation of compression waves transmitted to the substrate. ${ }^{21}$ Determination and interpretation of the morphological response to high strain rates are necessary for fundamental understanding of the performance. A number of studies have been carried out to measure the viscoelastic properties of PU: Boyce and co-workers ${ }^{7,8}$ and Zhao et al. ${ }^{9}$ conducted split Hopkinson bar measurements at compressive strain rates as high as $10^{4} \mathrm{~s}^{-1}$ on the material of interest herein. Jiao et al. ${ }^{6}$ used a pressure-shear impact plate to measure the response at shear rates between $10^{5}$ and $10^{6} \mathrm{~s}^{-1}$. The segmental dynamics of this PU were characterized by dielectric spectros-

\footnotetext{
* Corresponding author. E-mail: mike.roland@nrl.navy.mil.

George Mason University.

US Naval Research Laboratory.

$\S$ National Institute of Standards and Technology.

"Northboro Research and Development Center.

${ }^{\perp}$ University of Delaware.
}

copy at rates exceeding $10^{6} \mathrm{~s}^{-12}$ and at hydrostatic pressures approaching $1 \mathrm{GPa}^{4}$ Using a homemade tensile tester, ${ }^{22}$ we previously measured the stress-strain response of the PU at extensional strain rates, $\dot{\varepsilon}$, up to $\sim 600 \mathrm{~s}^{-1}$. $^{3}$

In this work we characterize the morphology of the PU from the temperature dependence of the small-angle X-ray scattering (SAXS) and then study the effects of tensile deformation through failure on the morphology by measuring SAXS on samples that had been stretched over a range of rates up to $830 \mathrm{~s}^{-1}$. Previous studies of this type were limited to quasi-statically deformed segmented block copolymers. ${ }^{23-26}$ We also measure the smallstrain dynamic modulus of PU across a temperature range spanning the soft segment glass transition. These experiments were motivated by recent work, ${ }^{9}$ suggesting that this PU conforms to the time-temperature superposition principle, ${ }^{27}$ with the resulting master curves used in modeling the impact response of the PU. ${ }^{5}$ Such superpositioning is surprising, since beyond the general breakdown of superpositioning for amorphous polymers in the softening zone (as known for polystyrene, ${ }^{28,29}$ poly(vinyl acetate), ${ }^{30}$ poly(propylene glycol), ${ }^{31}$ poly(phenylmethylsiloxane) ${ }^{32}$ polybutadiene, ${ }^{33,34}$ polyisobutylene, ${ }^{35}$ atactic polypropylene, ${ }^{36}$ poly(alkyl glycidyl ether) ${ }^{37}$ polypropylene,${ }^{38}$ and polyisoprene ${ }^{39}$ ), block copolymers in the ordered state are expected to be especially thermorheologically complex due to changes in their phase morphology with temperature. ${ }^{40}$ Finally, since annealing is an obvious route to refine the morphology of block copolymers, we study the interplay between morphology and rheology by measuring the temperature-dependent modulus of the PU at low frequency and compare it to the response of a specimen annealed between the order-disorder transition temperature and the melting point of the hard segments. 


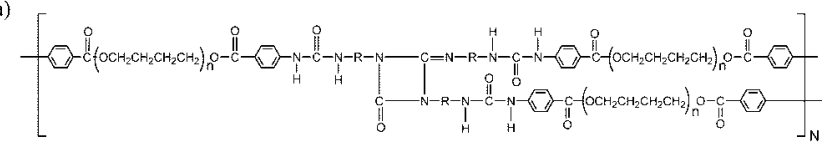

(b)<smiles></smiles>

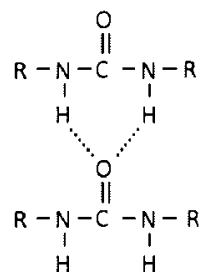

Figure 1. (a) Polyurea obtained from reaction of carbodiimide and diisocyanate adduct with poly(tetramethylene oxide-di- $p$-aminobenzoate), where $\mathrm{R}$ represents diphenylmethane. The nominal degree of polymerization of the poly(tetramethylene oxide) was 13 . The hard segment (derived from diisocyanate) is in the middle of the molecule. (b) Structures resulting from hydrogen bonding between amine and carbonyl groups.

\section{Experimental Section ${ }^{41}$}

Polyurea film was prepared by reacting four parts of poly(tetramethylene oxide-di-p-aminobenzoate) (Versalink P-1000; Air Products) with 1 part of a polycarbodiimide-modified diphenylmethane diisocyanate (Isonate $143 \mathrm{~L}$; Dow Chemical). The reaction is carried out by mixing the two liquids in the nozzle of an applicator at room temperature, with the liquid mixture then sprayed onto a substrate. The diamine and diisocyanate undergo rapid linear polycondensation ${ }^{42}$ to yield a microphase-separated block copolymer having $19.8 \%$ hard segments by mass (Figure 1 ). The cast material was used following at least 30 days annealing at ambient temperature. For one experiment the crystallinity was enhanced by annealing a sample at $175{ }^{\circ} \mathrm{C}$ for $30 \mathrm{~min}$ prior to quenching by immersion in liquid nitrogen.

Differential scanning calorimetry (DSC) using a TA DSC Q100 was carried out by heating at $10 \mathrm{~K} / \mathrm{min}$. DSC of the material showed the soft segment glass transition at $-62{ }^{\circ} \mathrm{C}$, an order-disorder transition centered at $141{ }^{\circ} \mathrm{C}\left(T_{\mathrm{ODT}}\right)$ with a spread of $27^{\circ} \mathrm{C}$, and the hard segment melting temperature at $194{ }^{\circ} \mathrm{C}$ (width $\sim 19^{\circ} \mathrm{C}$ ). The identification of the various thermal transitions was corroborated from the temperature-dependent SAXS.

Isothermal oscillatory shear measurements on rectangular strips (40 $\mathrm{mm}$ long, $10 \mathrm{~mm}$ wide and $2 \mathrm{~mm}$ thick) were conducted with two rheometers: a TA Instruments ARES and an Anton Paar MCR301. The measurement frequencies were in the range from $10^{-3}$ to $10^{2} \mathrm{rad} / \mathrm{s}$ at temperatures from $-63.5^{\circ} \mathrm{C}$ to ambient. Temperature sweep experiments were also carried out using a Bohlin VOR on rectangular strips heated at $3 \mathrm{deg} / \mathrm{min}$ from -69 and $175^{\circ} \mathrm{C}$; the frequency was $1.25 \mathrm{rad} / \mathrm{s}$. The high $\dot{\varepsilon}$ experiments were done on ASTM 4482 specimens using a custom-designed drop-weight apparatus. ${ }^{22}$ The stresses were determined from load cells attached to the sample grips and the strains from fiducial marks recorded with a high-speed camera (Vision Research Phantom 7; $10^{4} \mathrm{~s}^{-1}$ frame rate at 12 bit resolution). Conventional low-speed tests used an Instron 5500R with Wallace optical extensometer.

Two types of SAXS experiments were carried out. First, isothermal measurements were obtained on both undeformed PU and samples stretched to failure, the latter measured within $4 \mathrm{~h}$ of stretching. The X-ray source was molybdenum (wavelength $\lambda=$ $0.71 \AA ; \Delta \lambda / \lambda=10^{-2}$ ) with pinhole collimation and two-dimensional image plate detection. Sample thickness was $\sim 1.5 \mathrm{~mm}$. Data were typically acquired at three different locations in the gage region of each sample in order to verify that the deformation was uniform.

Second, temperature-dependent X-ray scattering data were acquired on the as-cast polyurea over the temperature range $25-225$ ${ }^{\circ} \mathrm{C}$ during both heating and cooling cycles. The X-ray source was copper $(\lambda=1.54 \AA$ ) with pinhole collimation and a Max-Flux optic.

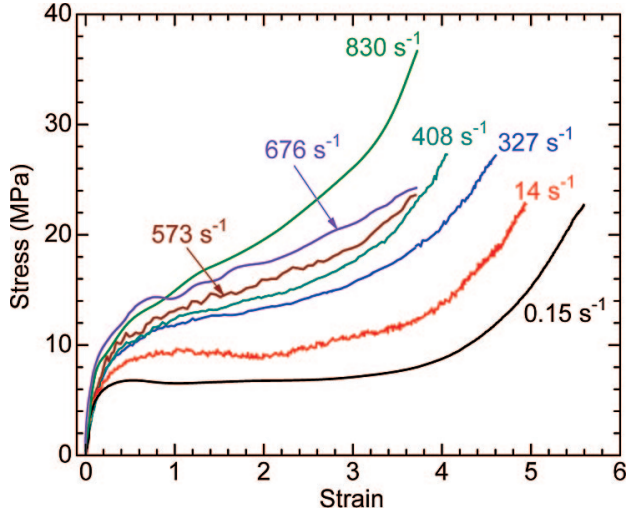

Figure 2. Engineering stress - strain curves at the indicated strain rates. Data at the two highest rates are new; the other data are from ref 3.

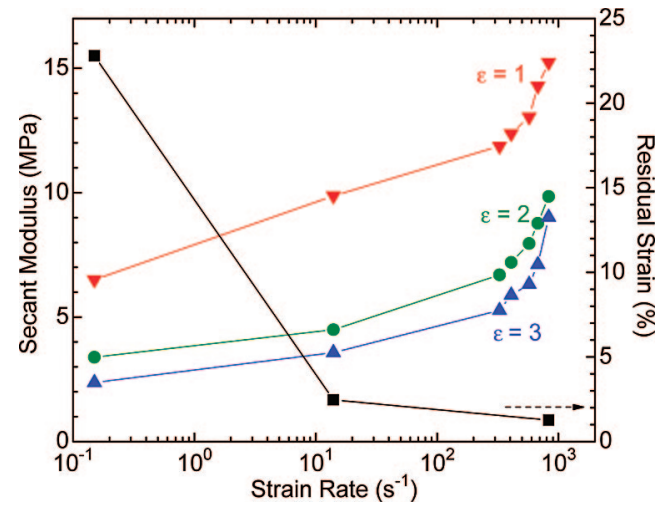

Figure 3. Ratio of the engineering stress to strain at the indicated strains as a function of extension rate, along with (right ordinate) the residual strain measured immediately after failure.

Two-dimensional scattering patterns were collected on a Gabriel multiwire area detector with a $120 \mathrm{~mm}$ diameter active area. The sample-to-detector distance was $1960 \mathrm{~mm}$. The sample was sandwiched between Kapton sheets, placed within an evacuated chamber/tube assembly, and maintained at the desired temperature by a modified Linkam THMS600 temperature control stage with liquid nitrogen cooling. The sample annealing times were $1 \mathrm{~h}$, followed by $3 \mathrm{~h}$ of data acquisition.

\section{Results and Discussion}

1. High Strain Rate Stress-Strain Curves and Residual Strain. In Figure 2 are the engineering stress-strain curves measured at strain rates spanning 3.7 decades (some of the data at lower $\dot{\varepsilon}$ were reported previously ${ }^{3}$ ). PU shows typical elastomeric behavior: increasing stiffness and strength and decreasing failure strain with increasing strain rate. ${ }^{43-45}$ The Young's modulus $E$ is high (130 MPa at $830 \mathrm{~s}^{-1}$ ); in Figure 3 the secant modulus at higher strains is plotted versus $\dot{\varepsilon}$. At ambient conditions the PU is in a microphase-separated state, with soft segments present as a matrix containing the hard segment aggregates, the latter functioning as cross-links and reinforcing filler for the deformable matrix. Around $300 \mathrm{~s}^{-1}$ there is noticeable stiffening of the PU. The unrecovered (residual) strain, $\varepsilon_{\mathrm{R}}$, was measured immediately after stretching to failure, although its time dependence was very weak. As seen in Figure $3, \varepsilon_{\mathrm{R}}$ is substantial at slow rates $(=23 \%$ for $\dot{\varepsilon}=0.15$ $\mathrm{s}^{-1}$ ) but becomes almost negligible at higher rates; e.g., $\varepsilon_{\mathrm{R}}=$ $1.3 \%$ for $\dot{\varepsilon}=830 \mathrm{~s}^{-1}$. (The difference is due entirely to the effect of rate. Although higher strains are expected to increase the mechanical hysteresis in polyurethanes and polyureas, ${ }^{20}$ the somewhat higher failure strain herein for slow rates exerts a negligible effect on $\varepsilon_{R}$.) From direct measurements of the local 


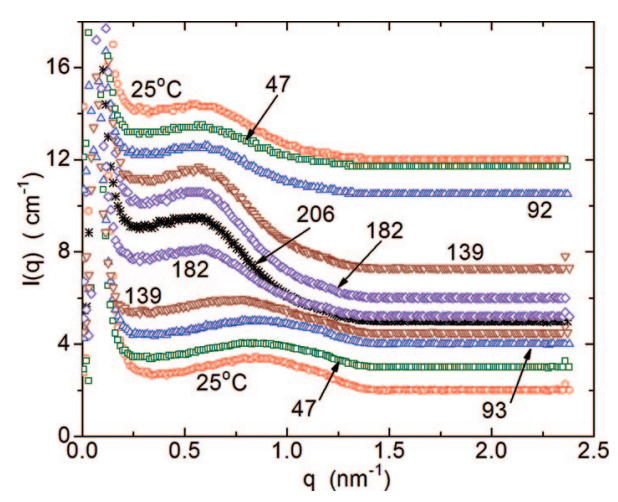

Figure 4. SAXS for the as-cast polyurea (data shifted vertically for clarity). The lower five curves were obtained during heating and the upper five during the subsequent cooling, with the temperatures (in ${ }^{\circ} \mathrm{C}$ ) as indicated.

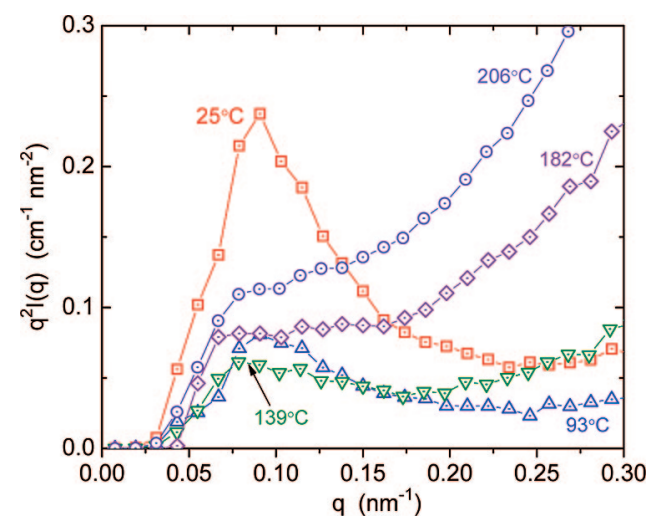

Figure 5. Lorentz-corrected scattering intensities in the low $q$ region for the as-cast PU after heating to the indicated temperatures (in ${ }^{\circ} \mathrm{C}$ ).

segmental dynamics in this $\mathrm{PU}^{2}$ the distribution of relaxation frequencies at room temperature is centered around $1 \mathrm{MHz}$, extending down to a few hundred hertz. Thus, at the high strain rates for which $\varepsilon_{\mathrm{R}} \sim 0$, the material is approaching the glass transition of the soft segments, as the inverse of the imposed rate reaches the inverse of the time scale for local segmental motion; $;^{2,7}$ consequently, deformation and morphological rearrangements become severely impeded.

2. Temperature-Dependent SAXS. The scattering intensities, $I(q, T)$ where $q=(4 \pi / \lambda) \sin (\theta / 2)$ and $\theta$ is the scattering angle, are shown for the as-cast polyurea in Figure 4 (data at different temperatures have been shifted vertically for clarity). At lower temperatures a peak at higher $q\left(\geq 0.5 \mathrm{~nm}^{-1}\right)$ changes minimally initially during heating but moves to lower angles over the range from 139 to $182{ }^{\circ} \mathrm{C}$. This reflects structural growth occurring at temperatures between $T_{\mathrm{ODT}}$ and $T_{\mathrm{m}}$. During subsequent cooling from 207 to $182{ }^{\circ} \mathrm{C}$, the peak shifts slightly to lower $q$ as the system reorders below $T_{\text {ODT. }}$. The peak position is then essentially invariant with further cooling to $25^{\circ} \mathrm{C}$.

A second SAXS peak is present at significantly lower $q(<0.3$ $\mathrm{nm}^{-1}$ ). It falls well within the angular resolution of the instrument and can be seen in the Lorentz-corrected scatting intensity $q^{2} I(q)$ shown in Figure 5. The peak persists up to 139 ${ }^{\circ} \mathrm{C}$, above which it appears as only a shoulder (see data at 182 and $206{ }^{\circ} \mathrm{C}$ in Figure 5); such a transition from a peak to a shoulder, seen previously in polyurethanes,${ }^{46-48}$ occurs as the system disorders above $T_{\mathrm{ODT}}$. Below $T_{\mathrm{ODT}}$ the hard segments are aggregated in domains, yielding a sharper interface between hard and soft segments than in the disordered state ${ }^{49}$ Of course, the soft segments in ordered segmented block copolymers can see both environments rich in themselves (in the matrix) and

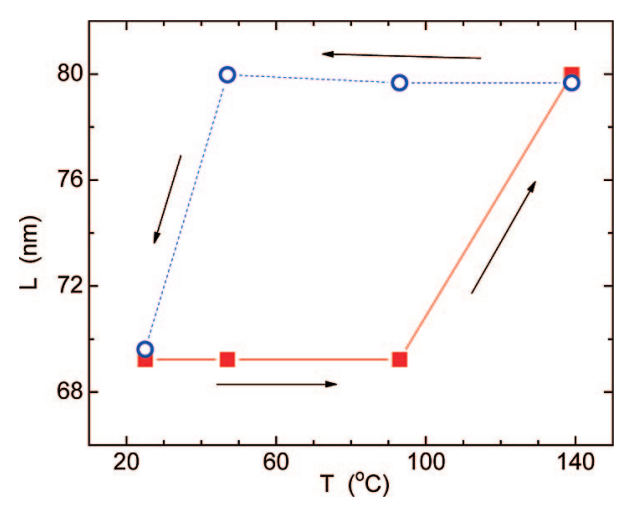

Figure 6. Variation of mean interdomain spacing with temperature in the as-cast PU during heating (squares) and subsequent cooling (circles).

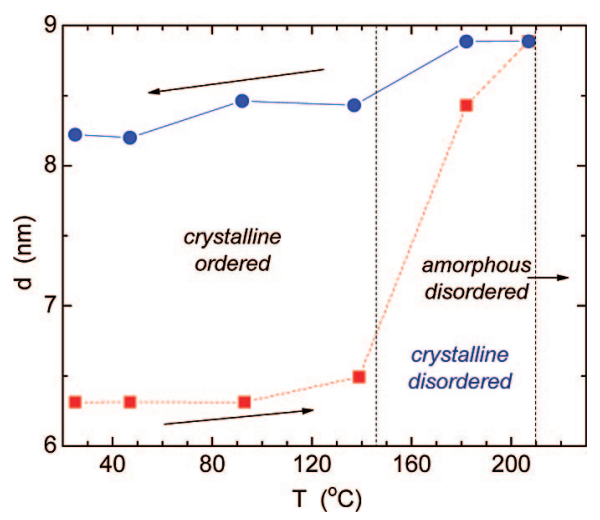

Figure 7. Variation of the long period in the as-cast PU during heating (squares) and subsequent cooling (circles). The approximate locations of the phase boundaries are indicated.

environments consisting of hard segments (at the interface between soft and hard segments). ${ }^{20}$

In systems with coupled ODT and crystallization, these two scattering peaks are expected. Since the mesophase is much larger than the crystal (lamellar) size, the peak from the hardsegment enriched domains falls at lower $q$ than the peak due to the semicrystalline hard segments. Upon recooling the system below $T_{\mathrm{ODT}}$, the lower $q$ peak reappears, signifying reordering. This assignment of the two SAXS peaks can be substantiated with two arguments. First, if the high angle peak were due to ordering, it would disappear when the system disorders upon heating above $T_{\mathrm{ODT}} \sim 182{ }^{\circ} \mathrm{C}$ (SAXS suggests that $139 \leq T_{\mathrm{ODT}}$ $\left({ }^{\circ} \mathrm{C}\right) \leq 182$ ), which does not occur (Figure 4). Instead, the peak shifts to lower $q$ due to crystal growth. Second, when the polyurea is heated above $T_{\mathrm{m}}$ to $225{ }^{\circ} \mathrm{C}$, the peak at high $q$ disappears, confirming its origin as hard-segment crystallites.

In Figures 6 and 7 we plot the average interdomain spacing $L\left(=2 \pi / q^{*}\right)$ and the long period $d\left(=2 \pi / q_{\max }\right)$ as a function of temperature, respectively. The parameters $q^{*}$ and $q_{\max }$ represent the scattering vector of the low and high angle peaks, respectively, determined from plots of $q^{2} I(q)$ vs $q$ (Figure 5). The figures show marked hysteresis, with higher values of $L$ and $d$ during cooling. Block copolymer thermodynamics theory ${ }^{50}$ estimates the radius of gyration $R_{\mathrm{g}}$ as $2 \leq q * R_{\mathrm{g}} \leq 3$, giving $R_{\mathrm{g}}$ to be $20-30 \mathrm{~nm}$ for this polyurea. Figure 7 shows a jump in $d$ between 139 and $182^{\circ} \mathrm{C}$, with similar discontinuities observed in related polymers. ${ }^{56}$ This jump suggests that crystal growth is retarded in the ordered state but is facilitated by disordering of the phase morphology. The three morphological regimes of the polyurea-crystalline ordered, crystalline disordered, and amorphous disordered-are denoted in Figure 7. 


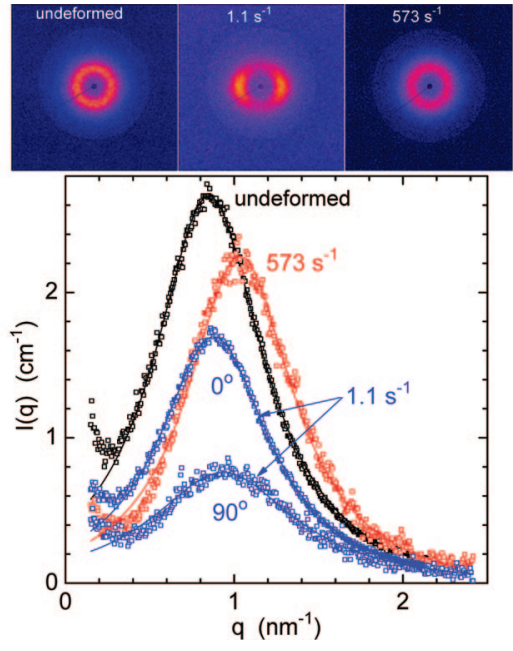

Figure 8. Top: scattering patterns (detector images) of undeformed polyurea and polyurea deformed at 1.1 and $573 \mathrm{~s}^{-1}$ (left to right). Bottom: corresponding scattering intensities, along with fitted shifted Lorentzian functions (smooth curves).

3. SAXS on Stretched Polyurea. In Figure 8, the 2D scattering patterns and $I(q)$ are shown for the PU initially and after straining to failure at $\dot{\varepsilon}=1.1$ and $573 \mathrm{~s}^{-1} . I(q)$ measured parallel and perpendicular to the principal stretch direction are shown separately. The peak in the vicinity of $q \sim 0.9 \mathrm{~nm}^{-1}$ is due to the semicrystalline hard domains. There is a second SAXS peak at smaller angle, arising from the phase-separated morphology. ${ }^{49}$ For slow deformation the scattering pattern is distinctly anisotropic, as the domains align themselves in the stretching direction. However, at higher strain rates this orientation is lost, as reflected in the isotropy of the scattering. Scattering data for other $\dot{\varepsilon}$ are qualitatively similar-anisotropic at slow to moderate rates and isotropic at high rates. This change from oriented to isotropic morphology is consistent with the decrease of $\varepsilon_{\mathrm{R}}$ with increasing strain rate (Figure 3).

An analysis of the SAXS data shows a similar dependence on strain rate. The long period was calculated using the Bragg formula, $d=2 \pi / q_{\max }$, where $q_{\max }$ is the scattering vector of the maximum in the Lorentz-corrected intensity scattering angle. ${ }^{51}$ The long period is a measure of the lamellar size (mean periodicity length). The scattering invariant, $Q$, was evaluated as $^{51}$

$$
Q=\frac{1}{2 \pi^{2}} \int_{0}^{\infty} q^{2} I(q) \mathrm{d} q
$$

although since the measurements did not extend to $q=0$ and $q=\infty, Q$ was estimated from integration between the experimental $q$ limits. The invariant depends on the phase contrast and concentration, so that it is sensitive to microphase mixing at the interfacial regions. The results for $d$ and $Q$ are plotted versus $\dot{\varepsilon}$ in Figure 9. Note that at the highest strain rates the deformation-induced changes in the structural parameters return to essentially the same values measured for the undeformed sample. This again shows that disruption of the domain morphology is only induced by deformation at slow or moderate strain rates.

From these results it is apparent that at slow strain rates the hard segment domains undergo reorganization in response to the stress, giving rise to permanent set and the anisotropic SAXS patterns. At larger $\dot{\varepsilon}$ the morphological response is too slow, so that the domain structure is unaffected. Under these conditions the hard domains function more effectively as a reinforcing filler, contributing to the strain hardening of the elastomer. The change in behavior is governed by the local segmental relaxation time

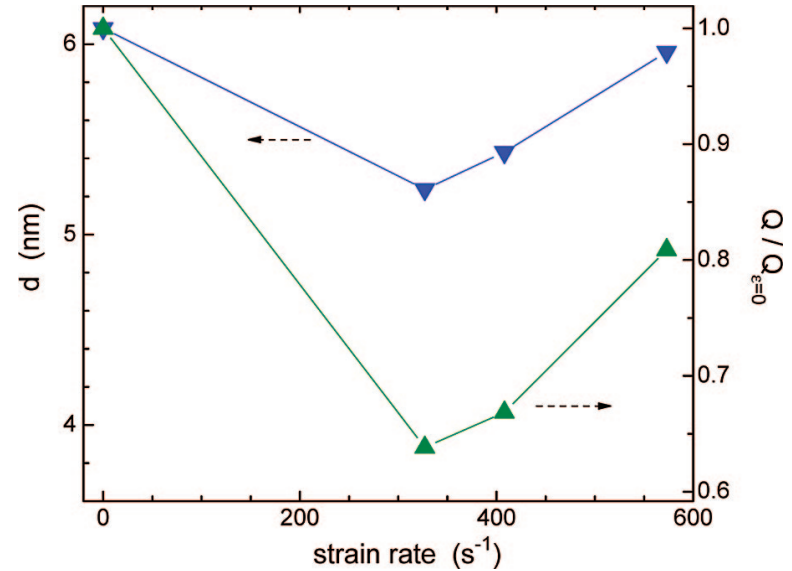

Figure 9. Variation with strain rate of the long period (inverted triangles) and scattering invariant (triangles), the latter normalized by the value for the unstretched sample.

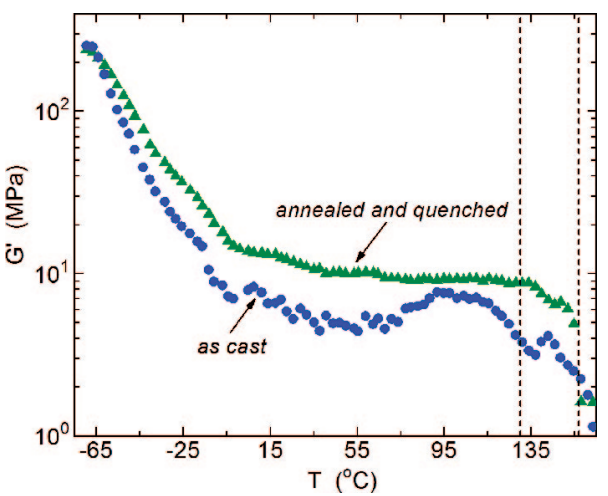

Figure 10. Dynamic storage modulus measured at $\omega=1.25 \mathrm{rad} / \mathrm{s}$ during heating at $3 \mathrm{deg} / \mathrm{min}$ for PU initially (circles) and after annealing at $175{ }^{\circ} \mathrm{C}$ followed by quenching (triangles). The range of the order-disorder transition measured calorimetrically is indicated by the vertical dashed lines.

of the soft segments at the (ambient) temperature of the experiments, $\sim 2 \mathrm{~ms}$, and thus is related to the approach to their glass transition zone at high strain rates.

4. Linear Viscoelasticity. The reaction of the isocyanate and diamine is very rapid, contributing to the complex, nonequilibrium morphology. This morphology can be refined by annealing, ${ }^{52,53}$ but only to a limited extent because of polydispersity of both the block length and the copolymer molecular weight. ${ }^{54}$ (The structure of block copolymers in the ordered state is often a thermodynamically metastable state..$^{55}$ ) Annealing at $175{ }^{\circ} \mathrm{C}$ (below the hard segment melting point, $T_{\mathrm{m}}=194{ }^{\circ} \mathrm{C}$, but above the $T_{\mathrm{ODT}}$ ) increases the crystallinity since it is no longer impeded by the ordered structure. ${ }^{56}$ The effect on the shear storage modulus, $G^{\prime}$, is shown in Figure 10; there is about a 2-fold increase after annealing and quenching. The as-cast PU shows some stiffening between 75 and $125^{\circ} \mathrm{C}$ due to crystal growth during the measurement. Beginning around $131^{\circ} \mathrm{C}$ both samples show a decrease in $G^{\prime}$, as the effect of disordering overwhelms any effect of further crystallization. The range of the order-disorder transition is consistent with the results from DSC (indicated by the horizontal dashed lines in Figure 10). No data were obtained above $175{ }^{\circ} \mathrm{C}$ due to instrumental limitations, although marked softening of the material was obvious above the melting temperature of the hard segments.

Recently, master curves were reported for this same PU, obtained by time-temperature superpositioning of linear stress relaxation measurements over the range -49 to $22{ }^{\circ} \mathrm{C}$. ${ }^{9}$ The ostensibly successful superpositioning is surprising, since even 


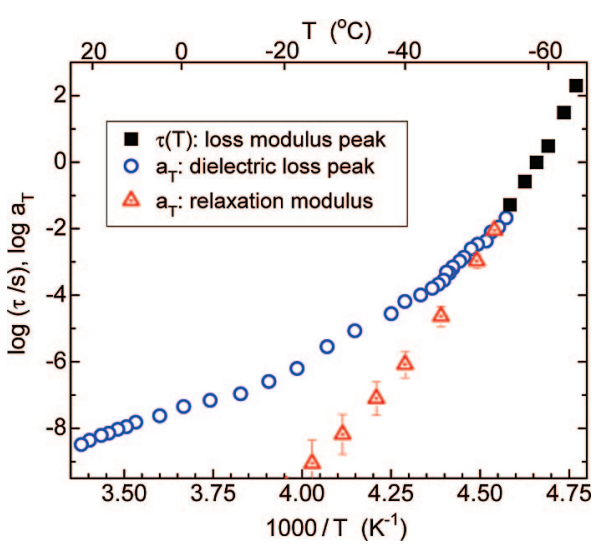

Figure 11. Local segmental relaxation times defined from the inverse frequency of the mechanical loss modulus (filled squares); shift factors for dielectric relaxation times (open circles) taken from ref 2; shift factors from mechanical relaxation (open triangles) taken from refs 5 and 9 .

homopolymers having an amorphous, homogeneous morphology exhibit thermorheological complexity in the softening zone. ${ }^{28-39}$ This complexity should be greater for the PU, since its microphase-separated morphology, crystallinity, and degree of hydrogen bonding are all temperature-dependent features that potentially contribute to deviations from time-temperature superpositioning. Thermorheological complexity due simply to the temperature dependence of the phase morphology has been demonstrated in styrene-isoprene block copolymers below the $T_{\text {ODT. }}{ }^{40}$

In Figure 11 the segmental relaxation times, defined as the inverse of the frequency of the maximum in the mechanical loss modulus, $\tau=1 / \omega_{\max }$, are plotted versus reciprocal temperature. Included in the figure are the dielectric relaxation times for the same material; ${ }^{2}$ these have been shifted to overlap the mechanical $\tau$ (dielectric relaxation times are always longer than the corresponding $\tau$ determined from the dynamic modulus ${ }^{57}$ ). The two data sets exhibit the same temperature dependence, consistent with both measurements probing the local segmental modes of the PU soft segments.

Also shown in Figure 11 are the time-temperature shift factors, $a_{T}$, for the PU determined from mechanical stress relaxation. ${ }^{9}$ These $a_{T}$ have a very different temperature dependence and deviate significantly from the other data. Note that the stress relaxation measurements were taken beginning at $0.01 \mathrm{~s},{ }^{9}$ which is an order of magnitude longer than the segmental relaxation time at even the lowest temperature of the experiments. At higher $T, \tau$ would be much shorter, so that the segmental process would have fully decayed before any stress relaxation was measured. Clearly, the transient experiments in ref 9 did not measure segmental relaxation of the PU soft segments.

Of course, in any temperature range over which the segmental and chain modes both contribute to the response, failure of time-temperature superpositioning is expected quite generally. Figure 12 shows the mechanical loss tangent measured at various temperatures, each shifted on the frequency axis to superpose the data. Comparing loss tangent curves is a sterner test of superpositioning, ${ }^{58-60}$ since unlike monotonic stress relaxation data, there is a peak in the loss tangent spectra and there is no vertical shifting (since any temperature or density effects on the storage and loss modulus cancel ${ }^{27}$ ). It is evident that the loss tangents for PU do not superpose in the transition zone. There is a systematic increase in magnitude with decreasing $T$, as the contribution of the segmental modes increases relative to that due to the chain modes with proximity to $T_{\mathrm{g}} .{ }^{39} \mathrm{Such}$

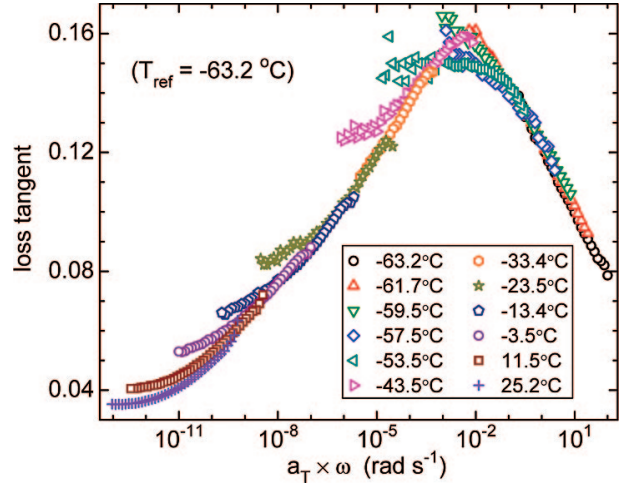

Figure 12. Mechanical loss tangent measured at the indicated temperatures, demonstrating the breakdown of time-temperature superpositioning in the softening zone. The data were shifted to superpose the position of the peak maxima; the latter decreases with increasing temperature.

thermorheological complexity is apparently not evident in the corresponding stress relaxation measurements. ${ }^{9}$

\section{Conclusions}

This work shows that the PU elastomer, which is known to convert to a glassy state under impact loading $\left(\dot{\varepsilon} \geq 10^{5} \mathrm{~s}^{-1}\right){ }^{2}$ responds differently to moderate strain rates of ca. $500 \mathrm{~s}^{-1}$ or less. Slow deformation disrupts the morphology, causing some interfacial mixing and measurable permanent set; however, at higher rates the initially unoriented structure is retained, with complete recovery from large deformation and isotropic SAXS. The complex, nonequilibrium nature of the PU (microphaseseparated domains with partially crystalline hard segments) is evident from temperature-dependent SAXS measurements in combination with DSC. These structural features contribute to thermorheologically complexity of the material's dynamic response. This complexity is expected for polymers in general but in particular for the polyurea given its morphology. The observed breakdown of time-temperature superpositioning, however, contradicts published master curves 9 for this same material. This is a significant point because the latter results have been used in modeling the mechanical properties of PU coatings intended for impact resistance. ${ }^{5}$ The mechanical relaxation times measured herein are consistent with dielectric relaxation measurements, extending the latter another 4 decades toward lower frequencies.

Acknowledgment. The work at the Naval Research Laboratory was supported by the Office of Naval Research. We thank David Owen (NSWC-Carderock) for preparing the polyurea, Howard Schrader (NRL) for assistance with the high strain rate experiments, and Radoslav Bogoslovov (NRL) for useful comments. The Rigaku $\mathrm{X}$-ray scattering instrument at the University of Delaware was purchased with funding from the Delaware Biotechnology Institute. We also thank Anton-Paar, USA (Reinhard Eberl, Gerd Langenbucher, Daniel Sanborn, Prajakta Kamerkar, and James Eickhoff), for on-site loan of the MCR-301 rheometer and Holly RicksLaskoski; (NRL), Manoj Kolel-Veetil (NRL), and Jack Douglas (NIST) for helpful discussions.

\section{References and Notes}

(1) Xue, Z.; Hutchinson, J. W. Mech. Mater. 2007, 39, 473.

(2) Bogoslovov, R. B.; Roland, C. M.; Gamache, R. M. Appl. Phys. Lett. 2007, 90, 221910.

(3) Roland, C. M.; Twigg, J. N.; Vu, Y.; Mott, P. H. Polymer 2007, 48, 574.

(4) Roland, C. M.; Casalini, R. Polymer 2007, 48, 5747.

(5) Amirkhizi, A. V.; Isaacs, J.; McGee, J.; Nemat-Nasser, S. Philos. Mag. 2006, 36, 5847. 
(6) Jiao, T.; Clifton, R. J.; Grunschel, S. In Shock Compression of Condensed Matter; Furnish, M., Elert, M., Russell, T. P., White, C. T., Eds.; American Institute of Physics: Melville, NY, 2006.

(7) Yi, J.; Boyce, M. C.; Lee, G. F.; Balizer, E. Polymer 2006, 47, 319.

(8) Sarva, S. S.; Deschanel, S.; Boyce, M.; Chen, W. Polymer 2007, 48, 2208.

(9) Zhao, J.; Knauss, W. G.; Ravichandran, G. Mech. Time-Depend. Mater. 2007, 11, 289.

(10) Chakkarapani, V.; Ravi-Chandar, K.; Liechti, K. M. J. Eng. Mater. Technol. 2006, 128, 489.

(11) Tekalur, S. A.; Shukla, A.; Shivakumar, K. Comp. Struct. 2008, 84, 271.

(12) Willkomm, W. R.; Chen, Z. S.; Macosko, C. W.; Gobran, D. A.; Thomas, E. L. Polym. Eng. Sci. 1988, 28, 888.

(13) Ryan, A. J.; Stanford, J. L.; Wilkinson, A. N. Polym. Bull. 1987, 18, 517.

(14) Ryan, A. J. Polymer 1990, 31, 707.

(15) Coleman, M. M.; Sobkowiak, M.; Pehlert, G. J.; Painter, P. C. Macromol. Chem. Phys. 1997, 198, 117.

(16) Mattia, J.; Painter, P. Macromolecules 2007, 40, 1546.

(17) Das, S.; Yilgor, I.; Yilgor, E.; Wilkes, G. L. Polymer 2008, 49, 174.

(18) Kaushiva, B. D.; Wilkes, G. L. Polymer 2000, 41, 6987.

(19) Chattopadhyay, D. K.; Raju, K.V.S.N. Prog. Polym. Sci. 2007, 32, 352.

(20) Wang, C. B.; Cooper, S. L. Macromolecules 1983, 16, 715.

(21) Bahei-El-Din, Y. A.; Yehia, A.; Dvorak, G. J. Mech. Adv. Mater. Struct. 2007, 14, 465.

(22) Mott, P. H.; Twigg, J. N.; Roland, D. F.; Schrader, H. S.; Pathak, J. A.; Roland, C. M. Rev. Sci. Instrum. 2007, 78, 045105.

(23) Versteegen, R. V.; Kleppinger, R.; Sijbesma, R. P.; Meijer, E. W. Macromolecules 2006, 39, 772.

(24) Curgul, S.; Yilgor, I.; Yilgor, E.; Erman, B.; Cakmak, M. Macromolecules 2004, 37, 8676.

(25) Yeh, F.; Hsiao, B. S.; Sauer, B. B.; Michel, S.; Siesler, H. W. Macromolecules 2003, 36, 1940.

(26) Koerner, H.; Kelley, J. J.; Vaia, R. A. Macromolecules 2008, 41, 4709.

(27) Ferry, J. D. Viscoelastic Properties of Polymers, 3rd ed.; Wiley: New York, 1980.

(28) Plazek, D. J. J. Phys. Chem. 1965, 6, 612.

(29) Pathak, J. A.; Colby, R. H.; Floudas, G.; Jerome, R. Macromolecules 1999, 32, 2553.

(30) Plazek, D. J. Polym. J 1980, 12, 43.

(31) Ngai, K. L.; Schonhals, A.; Schlosser, E. Macromolecules 1992, 25, 4915.

(32) Plazek, D. J.; Bero, C.; Neumeister, S.; Floudas, G.; Fytas, G.; Ngai, K. L. Colloid Polym. Sci. 1994, 272, 1430.

(33) Palade, L. I.; Verney, V.; Attane, P. Macromolecules 1995, 28, 7051
(34) Robertson, C. G.; Rademacher, C. M. Macromolecules 2004, 37, 10009.

(35) Plazek, D. J.; Chay, I.-C.; Ngai, K. L.; Roland, C. M. Macromolecules 1995, 28, 6432 .

(36) Santangelo, P. G.; Ngai, K. L.; Roland, C. M. Macromolecules 1996, 29, 3651 .

(37) Yamane, M.; Hirose, Y.; Adachi, K. Macromolecules 2005, 38, 10686.

(38) Roland, C. M.; Ngai, K. L.; Santangelo, P. G.; Qiu, X. H.; Ediger, M. D.; Plazek, D. J. Macromolecules 2001, 34, 6159.

(39) Santangelo, P. G.; Roland, C. M. Macromolecules 1998, 31, 3715.

(40) Han, C. D.; Kim, J. K. Polymer 1993, 34, 2533.

(41) Certain equipment, instruments or materials are identified in this paper in order to adequately specify the experimental details. Such identification does not imply recommendation by the National Institute of Standards and Technology nor does it imply the materials are necessarily the best available for the purpose.

(42) Odian, G. Principles of Polymerization, 4th ed.; Wiley-Interscience: Hoboken, NJ, 2004.

(43) Nielsen, L. E.; Landel, R. F. Mechanical Properties of Polymers and Composites, 2nd ed.; Marcel Dekker: New York, 1994.

(44) Chen, Z. S.; Yang, W. P.; Macosko, C. W. Rubber Chem. Technol. 1988, 61,86

(45) Ryan, A. J.; Stanford, J. L.; Birch, A. J. Polymer 1993, 34, 4874.

(46) Ryan, A. J.; Macosko, C. W.; Bras, W. Macromolecules 1992, 25 , 6277.

(47) Li, Y.; Gao, T.; Liu, J.; Linliu, K.; Desper, C. R.; Chu, B. Macromolecules 1992, 25, 7365.

(48) Li, Y.; Gao, T.; Chu, B. Macromolecules 1992, 25, 1737.

(49) Wilkes, G. L.; Abouzahr, S. Macromolecules 1981, 14, 458.

(50) Bates, F. S.; Frederickson, G. H. Annu. Rev. Phys. Chem. 1990, 41, 525.

(51) Glatter, O.; Kratky, O. Small Angle X-ray Scattering; Academic Press: London, 1982.

(52) Leung, L. M.; Koberstein, J. T. Macromolecules 1986, 19, 706.

(53) Koberstein, J. T.; Galambos, A. F. Macromolecules 1992, 25, 5618.

(54) Lehmann, S. A.; Meltzer, D. A.; Spiess, H. W. J. Polym. Sci., Polym. Phys. Ed. 1998, 36, 693.

(55) Hamley, I. W. The Physics of Block Copolymers; Oxford University Press: Oxford, UK, 1998

(56) Koberstein, J. T.; Russell, T. P. Macromolecules 1986, 19, 714.

(57) McCrum, N. G.; Read, B. E.; Williams, G. Anelastic and Dielectric Effects in Polymeric Solids; Dover Publications: New York, 1991.

(58) Plazek, D. J. J. Rheol. 1996, 40, 987.

(59) Cavaille, J. Y.; Corinne, J.; Perez, J.; Monnerie, L.; Johari, G. P. J. Polym. Sci., Polym. Phys. Ed. 1987, 25, 1235.

(60) Capodagli, J.; Lakes, R. Rheol. Acta, 2008, in press.

MA8011009 